\title{
Surface plasmon and photonic mode propagation in gold nanotubes with varying wall thickness
}

\author{
Jesse Kohl, ${ }^{1}$ Micha Fireman, ${ }^{2,}{ }^{*}$ and Deirdre M. O’Carroll ${ }^{1,3, \dagger}$ \\ ${ }^{1}$ Department of Materials Science and Engineering, Rutgers University, 607 Taylor Road, Piscataway, New Jersey 08854, USA \\ ${ }^{2}$ Thomas J. Watson Laboratory of Applied Physics, California Institute of Technology, Pasadena, California 91125, USA \\ ${ }^{3}$ Institute of Advanced Materials Devices and Nanotechnology and Department of Chemistry and Chemical Biology, Rutgers University, \\ 607 Taylor Road, Piscataway, New Jersey 08854, USA
}

(Received 9 September 2011; revised manuscript received 14 November 2011; published 5 December 2011)

\begin{abstract}
Gold nanotube arrays are synthesized with a range of wall thicknesses ( 15 to $>140 \mathrm{~nm}$ ) and inner diameters of $\sim 200 \mathrm{~nm}$ using a hard-template method. A red spectral shift $(>0.39 \mathrm{eV})$ with decreasing wall thickness is observed in dark-field spectra of nanotube arrays and single nanowire/nanotube heterostructures. Finite-difference-timedomain simulations show that nanotubes in this size regime support propagating surface plasmon modes as well as surface plasmon ring resonances at visible wavelengths (the latter is observed only for excitation directions normal to the nanotube long axis with transverse polarization). The energy of the surface plasmon modes decreases with decreasing wall thickness and is attributed to an increase in mode coupling between propagating modes in the nanotube core and outer surface and the circumference dependence of ring resonances. Surface plasmon mode propagation lengths for thicker-walled tubes increase by a factor of $\sim 2$ at longer wavelengths $(>700 \mathrm{~nm})$, where ohmic losses in the metal are low, but thinner-walled tubes $(30 \mathrm{~nm})$ exhibit a more significant increase in surface plasmon propagation length (by a factor of more than four) at longer wavelengths. Additionally, nanotubes in this size regime support a photonic mode in their core, which does not change in energy with changing wall thickness. However, photonic mode propagation length is found to decrease for optically thin walls. Finally, correlations are made between the experimentally observed changes in dark-field spectra and the changes in surface plasmon mode properties observed in simulations for the various gold nanotube wall thicknesses and excitation conditions.
\end{abstract}

DOI: $10.1103 /$ PhysRevB.84.235118

PACS number(s): 73.20.Mf, 78.67.Ch, 81.05.Bx, 81.07.-b

\section{INTRODUCTION}

Nanotubes, like nanowires, exhibit desirable properties for a host of applications, including chemical sensing, subwavelength light-emitting diodes, and waveguides due to their anisotropic cylindrical shape - exhibiting nanoscopic diameters but macroscopic lengths. ${ }^{1}$ The advantage of metallic nanotubes compared with nanowires, in terms of sensing capability, is their greater surface-area-to-volume ratio and evanescent surface plasmon mode propagation at the metalanalyte interface on both the inner and outer tube surfaces. ${ }^{2}$ Sensing enhancement as a function of increased surfacearea-to-volume ratio has been observed previously when comparing solid gold nanospheres to gold nanoshells, with the latter showing a greater wavelength shift per unit change in surrounding refractive index. ${ }^{3,4}$

The resonant properties of noble metal nanotubes, like nanoshells, can be tuned through control of inner diameter and wall thickness. In the case of gold nanotubes (AuNTs), a pronounced red shift has been observed with decreasing wall thickness (WT) and increasing inner diameter (ID) (for WT ranging from $4-30 \mathrm{~nm}$ and ID ranging from $12-30 \mathrm{~nm}){ }^{5-7} \mathrm{It}$ is noteworthy that, while for tubular and core-shell structures an apparent red spectral shift is observed as a function of decreasing shell thickness, the opposite trend is observed in solid nanoparticle systems (spheres and rods), where a red shift and spectral broadening occur as a function of increasing size. ${ }^{8}$ Therefore, core-shell and tubular systems can be considered unique in their plasmonic response relative to solid geometries.

Prior work on fabrication and optical modeling of AuNT and core-shell structures has been focused on geometries with
IDs and WTs much smaller than the wavelength of light $(<30 \mathrm{~nm})$ and WT $\leqslant 42 \mathrm{~nm}^{5-7}$ In that size regime, intrawall electric-field coupling (i.e. coupling between the inner and outer surface of the wall) is strong, and surface plasmon hybridization models can be applied (quasistatic regime) ${ }^{9-13}$ For larger ID structures (ID $>100 \mathrm{~nm}$ ) where WT is still $<50 \mathrm{~nm}$ but intrawall coupling is weak, the effects of changing wall thickness are likely to dominate. In that size regime, the structure becomes more comparable to insulator-metalinsulator (IMI) slab waveguides, supporting both propagating surface plasmon polariton (SPP) and photonic modes. ${ }^{14}$

In this work, arrays of large 200-nm ID, 2 to $3-\mu$ m-long AuNTs were synthesized to study the optical properties and SPP mode propagation as a function of wall thickness and excitation direction. Dark-field scattered-light spectra of both vertical arrays of AuNTs and a single nanowire/nanotube (NW/NT) heterostructure were obtained. Optical measurements of single structures allow for more accurate assessment of the spectral characteristics without inhomogeneous broadening resulting from variance in geometry (e.g. length, diameter, wall thickness) of the synthesized AuNTs.

Simulations carried out by finite-difference-time-domain (FDTD) were conducted to identify and understand the modes supported by AuNTs with different wall thicknesses (varying from 30 to $140 \mathrm{~nm}$ ). These simulations demonstrate that large 200-nm ID AuNTs support three different mode types that depend on excitation direction and polarization: (1) when excitation is incident at the AuNT end, which we refer to as end-on excitation, propagating SPP modes are excited along the length of the nanotube walls, analogous to that of planar metal films and IMI waveguides. ${ }^{14}$ (2) In addition to the 
propagating SPP mode, a photonic mode is excited in the core of the nanotube. Similarly, for excitation normal to the nanotube long axis (which we refer to as normal excitation) and polarized parallel to the nanotube long axis, both a propagating SPP mode and a photonic mode are also supported. Theoretical propagation length studies for both the SPP and photonic modes were carried out. (3) For excitation normal to the nanotube and polarized transverse to the long axis, surface plasmon ring resonances occur at visible wavelengths. The ring resonances occur radially about the tube surface to form standing waves with electric field patterns that depend on the AuNT's circumference. We relate the modes observed theoretically to those detected experimentally to explain the electromagnetic response of the nanotubes as a function of decreasing wall thickness.

These results demonstrate that nanotubes that support surface plasmons have potential for use as both plasmonic waveguides and nanoscale optical resonators. Additionally, the relatively large inner diameter of the AuNTs studied here could facilitate filling of the core with a suitable dye or semiconductor material to introduce gain to the waveguide or cavity. Such waveguides and cavities may be a viable replacement for more conventional surface plasmon waveguide/cavity devices since the SPP field can be excited directly by tapered fiber facets. ${ }^{15-23}$

\section{METHODS}

\section{A. Electrodeposition conditions}

To fabricate AuNTs, firstly, a 400-nm-thick nickel film was thermally evaporated onto one side of a nanoporous anodic alumina (AAO) template (200-nm nominal pore diameter, 60- $\mu \mathrm{m}$-thick, 13-mm total diameter; Anodisc 13, Whatman Inc.) to serve as the working electrode contact to the template pores. The AAO template was then placed in a homemade polyethylene electrodeposition cell. A copper plate and copper tape were used to make electrical contact to the nickel-coated side of the AAO template when placed in the cell. Epoxy was employed at the edges of the AAO template to ensure that only the exposed pores of the template were in direct contact with the electrolyte and to prevent any leakage of the electrolyte from the electrodeposition cell. The electrodeposition cell was then placed in a beaker with a water level the same height as the electrolyte within the electrodeposition cell to facilitate ultrasonication. An alligator clip connected to a 10-cm length of steel wire immersed in the electrolyte served as the positive counter electrode; using an appropriate retort stand, the tip of the steel wire was submerged in the electrolyte.

To electrodeposit sacrificial nickel nanowires, $\sim 5 \mathrm{~mL}$ of a nickel plating solution (semi-bright finish; Alfa Aesar) was added to the electrodeposition cell and $-1 \mathrm{~mA}$ constant current was applied to the working electrode for 2 hours. The entire process was carried out under ultrasonication to ensure that a regular distribution of nanowires was grown across the entire template (Model 2510 Ultrasonic Cleaner, Branson). Scanning electron microscopy (SEM) data revealed that the length of the nickel nanowires ranged from 5 to $10 \mu \mathrm{m}$, and diameters corresponded to those of the AAO pores (200-nm nominal pore diameter; 240-nm average measured diameter). Following a pore-widening step using an $8.5 \%$ wt. solution of $\mathrm{H}_{3} \mathrm{PO}_{4}$ in deionized water (duration of 60,30 , or $15 \mathrm{~min}$.), gold was electrodeposited onto the nickel nanowires using approximately $1 \mathrm{~mL}$ of gold plating solution (metal content $25 \mathrm{~g} / \mathrm{L}$; Alfa Aesar) and applying $-1 \mathrm{~mA}$ constant current to the working electrode for 15 to $20 \mathrm{~min}$ to form goldcoated nickel nanowires. Although electrodepositions were run at a constant applied current of $-1 \mathrm{~mA}$ (6430 subfemtoamp source meter, Keithley Instruments, Inc.), the exact resistance of the deposition area varied from deposition to deposition due to the geometry of the nanoporous alumina template and slight differences in coverage of the insulating epoxy. The most uniform electrodepositions occurred at voltages no greater than 4 volts, often in the range of 3 to 4 volts.

\section{B. Sample preparation}

In order to prepare samples for SEM imaging and optical characterization, the filled AAO template sample was removed from the electrodeposition cell, mounted on a carbon pad (with nickel working electrode side facing upwards), and subsequently, the nickel working electrode, sacrificial nickel nanowire cores, and AAO were etched. To etch the nickel working electrode, along with the sacrificial nickel nanowire cores, the sample was submerged in a ferric chloride solution (Nickel Etchant, Type 1, Transene Company Inc.) for $1 \mathrm{hr}$ to expose the gold nanotubes embedded in the AAO. To etch the AAO, the sample was submerged in a solution of $3 \mathrm{M} \mathrm{NaOH}$ for $1 \mathrm{hr}$. The remaining gold nanotube array was then rinsed with deionized water and allowed to dry prior to characterization.

\section{Scattered-light spectroscopy of gold nanotubes}

A Zeiss Axio Observer Z1 was used to acquire bright-field and dark-field reflected light images and spectra of gold nanotube arrays. Here, $50 \times$ and $100 \times$ dark-field objectives were used to collect light from a $\sim 30-\times 60-\mu \mathrm{m}$ region of a gold nanotube array. A $100-\mathrm{W}$ halogen lamp was used as the light source for bright-field and dark-field microscopy. A darkfield microscope setup was employed to acquire scattered-light spectra of vertically oriented gold nanotube arrays. Under this configuration, unpolarized light from a halogen lamp was directed by an annular reflector and dark-field objective to obliquely illuminate the sample. Only scattered-light was collected by the central path of the reflector/objective and sent to an imaging spectrometer. For single nanowire/nanostructure scattered-light spectroscopy, the nanostructures were dispersed from arrays in methanol (by ultrasonication) and drop deposited onto glass cover slips. The imaging spectrometer was employed to acquire spectra from regions along the length of individual nanowire/nanotube heterostructures with a spatial resolution of $\sim 500 \mathrm{~nm}$. All scattered-light spectra were normalized relative to the lamp spectrum.

\section{Electromagnetic simulations}

To identify the modes which can be supported by the nanotubes and understand the nature of their propagation as a function of wall thickness, FDTD simulations of single AuNTs were carried out, with WT of 30, 60, and $140 \mathrm{~nm}$ and fixed ID of $200 \mathrm{~nm}$, under broadband (450-850 nm), planewave excitation with FDTD Solutions software (Lumerical 
Solutions, Inc.). The dispersive dielectric constants of gold used for simulations were taken from the Handbook of Optical Constants of Solids, edited by Palik (Academic Press, New York, 1985). A fine mesh size of $5 \mathrm{~nm}$ in the radial direction at the metal shell (at least five mesh cells thick) was employed, in order to accurately simulate the optical interaction. A chromium cylinder of $100 \mathrm{~nm}$ in length and equivalent inner and outer diameter to the corresponding simulated gold nanotube was placed at the opposite end of the gold nanotube to the plane wave source in order to mitigate back reflection of the propagating surface plasmon mode when extracting surface plasmon propagation length values.

\section{RESULTS}

Gold nanotube fabrication was carried out by a hardtemplate method, which employed sacrificial nickel nanowires as scaffolds onto which gold was electrochemically deposited. The fabrication steps are illustrated in Fig. 1(a) (see Methods). The first step required thermally evaporating $400 \mathrm{~nm}$ of nickel onto the back side of a nanoporous anodic alumina (AAO) template to act as the working electrode for subsequent nickel nanowire deposition. The next step exposed the top of the template to a dilute $8.5 \%$ wt. solution of $\mathrm{H}_{3} \mathrm{PO}_{4}$ under ultrasonication for durations of 60,30 , or $15 \mathrm{~min}$ in order to widen the template pores. After solvent rinsing, gold was then electrodeposited at $-1 \mathrm{~mA}$ for 15 to 20 minutes. After gold deposition, the sample was inverted, and the nickel cores and back nickel contact film were etched in a solution of $\mathrm{FeCl}_{3}$. Finally, the remaining AAO was removed in $\mathrm{NaOH}$ solution. This approach yielded vertically oriented arrays of $\sim 10^{8}$ gold nanotubes over an area of $\sim 30 \mathrm{~mm}^{2}$.

The scanning electron microscopy (SEM) images in Fig. 1 show that the duration of the pore-widening step had a direct effect on wall thickness and, consequently, scatteredlight spectra of the AuNTs. For longer pore-widening times (60 $\mathrm{min}$ ), which resulted in an average tube wall thickness of $136 \pm 36 \mathrm{~nm}$ [Fig. 1(b)], it is likely that the pores were etched all the way through to the nickel back working electrode contact, and as a result, gold electrodeposition may have occurred at the base of the sacrificial nickel nanowires. For shorter pore-widening times ( 30 and $15 \mathrm{~min}$ ), which resulted in average tube wall thicknesses of $55 \pm 18$ [Fig. 1(c)] and $29 \pm 8$ $\mathrm{nm}$ [Fig. 1(d)], respectively, the gold nanotube ends appeared to taper to very thin wall thicknesses as is evident from the flaky and flexible appearance [Fig. 1(d)], suggesting that pore widening did not occur uniformly along the length of the sacrificial nickel nanowires. Scattered-light spectra collected from four different regions of a vertically oriented array of nanotubes exhibited a broad peak at $657 \pm 14 \mathrm{~nm}(60$-min sample; using a dark-field objective in reflection mode under broadband, unpolarized halogen illumination of the nanotube ends). For the 30-min sample, scattered-light spectra exhibited a peak position of $676 \pm 63 \mathrm{~nm}$. The shortest pore-widening time $(15 \mathrm{~min})$ resulted in the most repeatable spectra with a peak position of $685 \pm 2 \mathrm{~nm}$ averaged over four different regions of the sample. The standard deviation in the peak position values was attributed to a distribution in the wall thicknesses and gold nanotube diameters across each sample. (a)
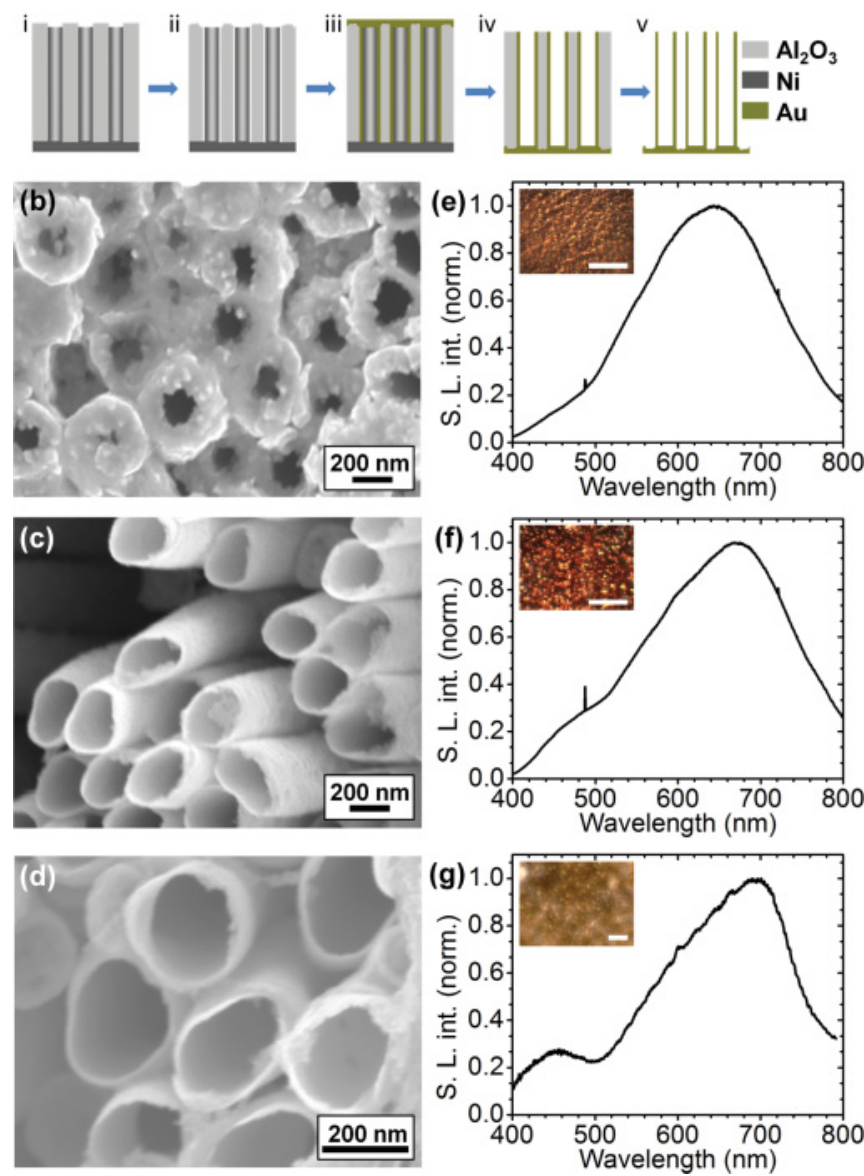

FIG. 1. (Color online) (a) Schematic showing the gold nanotube growth process [electrodeposition and pore-widening steps (i), (ii), and (iii) carried out under ultrasonication]. (b)-(d): SEM images of gold nanotubes deposited after AAO pore widening in $\mathrm{H}_{3} \mathrm{PO}_{4}$ for 60,30 , and 15 minutes, respectively. (e)-(g): Dark-field spectra, using unpolarized, end-on halogen lamp excitation, acquired from the samples imaged in (b)-(d), respectively. Top left corner insets in (e) to (g): True-color dark-field images of the nanotube arrays; scale bars are $25 \mu \mathrm{m}$

From this spectral data, it was apparent that the AuNTs preferentially scattered light of longer wavelengths for thinner wall thickness.

To more accurately measure and understand the trend in nanotube resonance spectra with wall thickness (without sampling multiple nanotubes with a distribution of sizes), scattered-light spectra were acquired from an individual nanowire/nanotube (NW/NT) heterostructure (dark-field reflection with broadband, unpolarized halogen illumination normal to the nanotube long axis) formed by over-depositing gold on the sacrificial nickel nanowires. Spectra recorded from the heterostructure at specific points along the structure [denoted by the colored Roman numerals in Fig. 2(d)] show that the resonance behavior red shifts from the nanowire to nanotube. Figure 2(a) shows a peak maximum for the nanowire end of the heterostructure at $600 \mathrm{~nm}$ and a red shift by more than $0.39 \mathrm{eV}(140 \mathrm{~nm})$ along the tube region. This shift in plasmon resonance is apparent in the reflected-light brightfield and dark-field true-color images of the heterostructure 

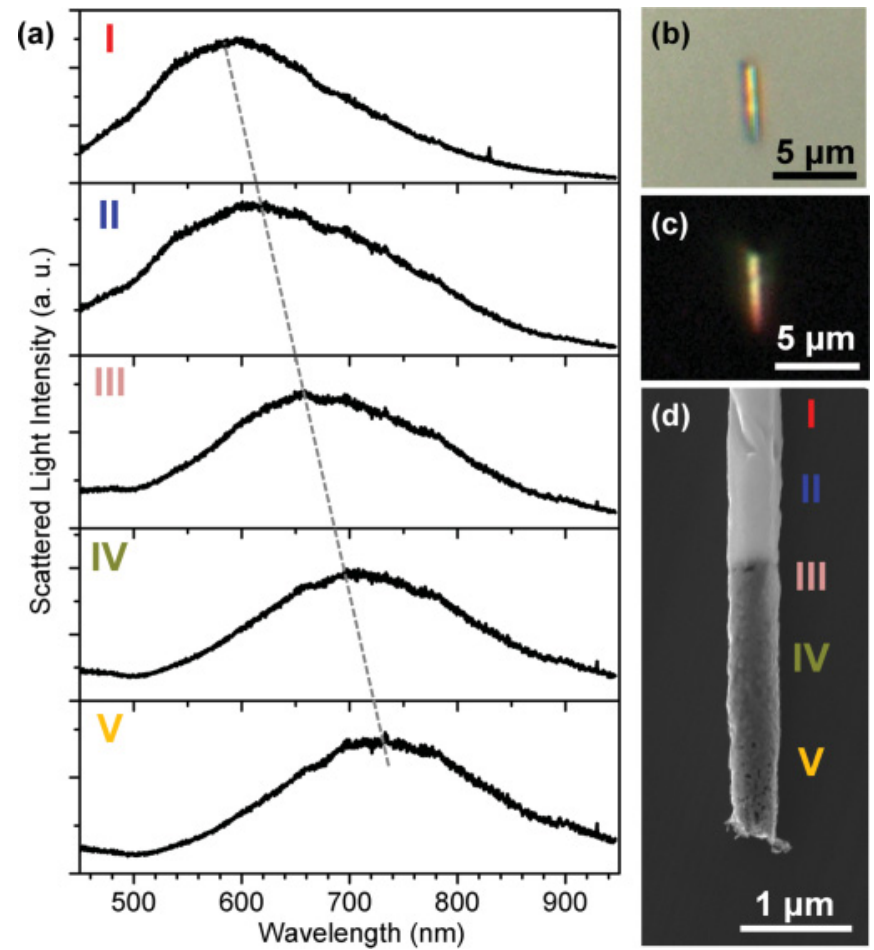

FIG. 2. (Color online) (a) Dark-field spectra using unpolarized, normally incident, halogen lamp excitation, acquired along the length of a single, nanotube/nanowire heterostructure [regions where the spectra were acquired from are indicated in with Roman numerals (d)]. Progressing downward along the structure, a red shift in resonance is evident as it becomes more tube-like. (b) Bright-field and (c) dark-field true-color optical images of the heterostructure. (d) SEM image of the NW/NT region of the structure.

[Figs. 2(b) and 2(c), respectively]. Clearly, the tube region (i.e. the lower $2.5 \mu \mathrm{m}$ of the heterostructure) in the bright-field reflected-light image is blue/green in color vs the wire region, which appears yellow. In the dark field image, the tube region scatters red light more effectively than the nanowire region.

The change in contrast along the nanotube length in the SEM image of the heterostructure [Fig. 2(d)] suggested that the wall thickness of the tube decreased towards the tip, where it became flaky and ultimately discontinuous due to the extreme thinness. Others have reported similar limitations in the thinness for which continuous gold shells can be fabricated. ${ }^{24}$ Therefore, the red shift of scattered-light spectra observed along the length of the nanotube section of the heterostructure was attributed to the decreasing wall thickness. These findings corroborate the red shift observed for the three nanotube arrays of different average wall thicknesses (Fig. 1).

3-dimensional FDTD simulations of AuNTs were carried out under end-on excitation, to resemble the excitation scheme employed to excite the large area AuNT arrays in the experimental data of Fig. 1 (nanotube length $=20 \mu \mathrm{m}$ in simulations), and under normal excitation conditions in analogy to the excitation scheme used to excite the single NW/NT heterostructure in Fig. 2 (nanotube length $=1 \mu \mathrm{m}$ ). For normal excitation, simulations were carried out for both excitation polarization parallel (i.e. axially polarized) and perpendicular (i.e. transversely polarized) to the nanotube
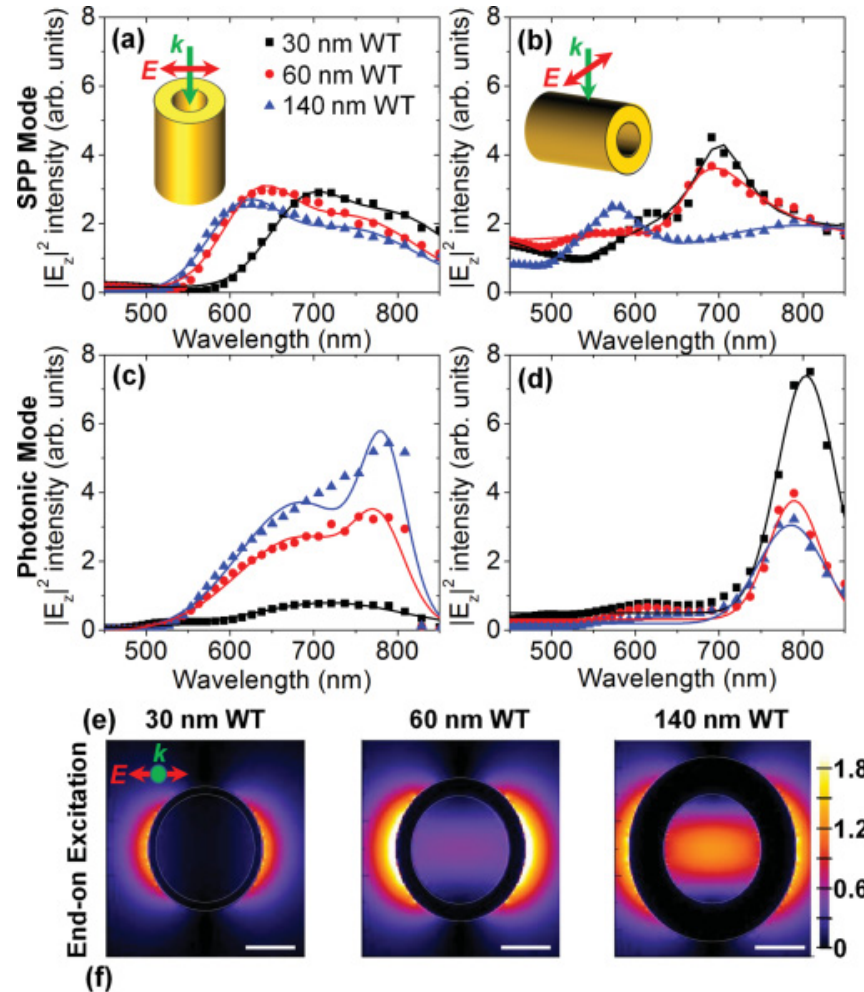

$140 \mathrm{~nm}$ WT
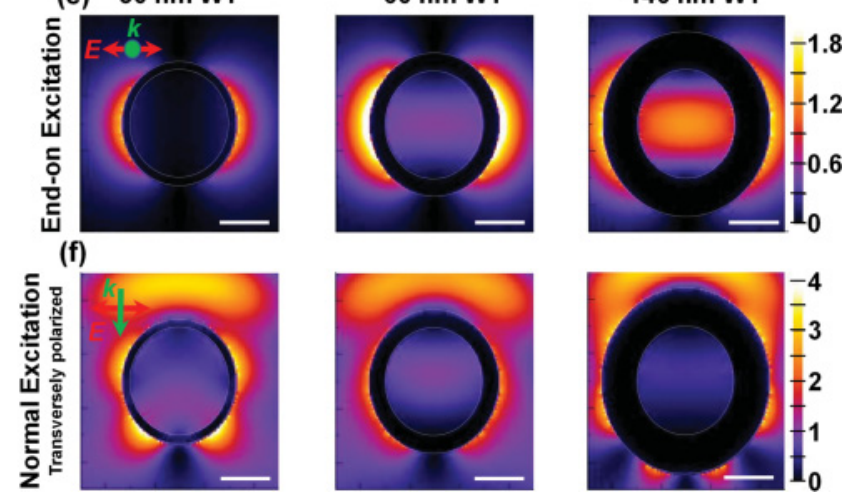

FIG. 3. (Color online) Electric-field intensity vs wavelength spectra for (a) and (b) the SPP, and (c) and (d) the photonic modes for (a) and (c) end-on excitation, and (b) and (d) transversely polarized normal excitation (spectra fitted with a double Gaussian). Inset in plots (a) and (b) are schematic representations of the end-on and normal excitation schemes, respectively. Green/gray arrows indicate excitation direction $(k)$ and red/dark gray arrows indicate the polarization direction $(E)$. Radial 2-D electric-field intensity profiles of (simulated AuNTs of 30-, 60-, and 140-nm WT under (e) end-on and (f) transversely polarized normal excitation at wavelengths corresponding to the maximum intensity in the corresponding SPP spectra shown in (a) and (b). Scale bars $=150 \mathrm{~nm}$; color bars in (e) and (f) represent electric-field intensity.

long axis, since an unpolarized excitation source was not available. The simulated dimensions were based on average measurements from SEM images of the fabricated AuNT arrays for the three different pore-widening times (see Fig. 1). The ID of the grown AuNT arrays varied slightly over the three different etching times, but the ID was held constant in the simulations to more clearly study the relationship between the plasmonic and photonic mode propagation and tube wall thickness.

From FDTD data, it was apparent that the AuNTs support both plasmonic and photonic modes in the wavelength range $600-850 \mathrm{~nm}$ and most intensely above $660 \mathrm{~nm}$. Electric-field intensity vs wavelength spectra for SPP modes propagating at the AuNT surface [Figs. 3(a) and 3(b)] and photonic modes in the nanotube core [Figs. 3(c) and 3(d)] were obtained to 
determine the wavelength at which the peak electric-field intensity occurred for the two mode types. SPP and photonic mode spectra were produced by averaging 20 points along a line scan $25 \mathrm{~nm}$ above the outer surface and along the central axis of the simulated tube, respectively, for both end-on [Figs. 3(a) and 3(c)] and normal [Figs. 3(b) and 3(d)] excitation conditions. For the SPP mode, peak electric field intensities (and mode quality factor, $Q=\lambda_{\text {res }} / \Delta \lambda$, where $\lambda_{\text {res }}$ is the resonance wavelength and $\Delta \lambda$ is the full-width-at-halfmaximum) occurred at wavelengths of 741, 688, and $678 \mathrm{~nm}$ under end-on excitation ( $Q$ values of 4, 3, and 3, respectively) and 708, 710, and $580 \mathrm{~nm}$ under transversely polarized normal excitation ( $Q$ values of 6,6, and 8, respectively) for AuNTs of 30-, 60-, and 140-nm WT, respectively. For AuNTs under axially polarized normal excitation, a propagating SPP mode and a photonic mode analogous to that observed when excited end-on were observed. Peak electric-field intensities for the simulated photonic mode occurred at wavelengths of 718 , 718 , and $729 \mathrm{~nm}$ under end-on excitation ( $Q$ values of 4 , 3 , and 4, respectively) and 804, 790, and $786 \mathrm{~nm}$ under transversely polarized normal excitation ( $Q$ values of 10,11 , and 9, respectively) for AuNTs of 30-, 60-, and 140-nm WT, respectively.

From the simulated spectral data, it was apparent that SPP mode spectra exhibited a red spectral shift with decreasing wall thickness, in qualitative agreement with experimentally observed data (see Figs. 1 and 2). A red shift of $0.16 \mathrm{eV}$ $(63 \mathrm{~nm})$ under end-on excitation and $0.39 \mathrm{eV}(128 \mathrm{~nm})$ under transversely polarized normal excitation was determined from the simulated data over the studied WT range (140 to $30 \mathrm{~nm})$. The red shift observed in the simulation results for the end-on excited AuNTs is two times greater in magnitude than the shift observed for the fabricated AuNT arrays under end-on excitation $[0.08 \mathrm{eV}(28 \mathrm{~nm})]$ over the same wall thicknesses range (Fig. 1). This disparity in the magnitude of the red shift is attributed to experimental variations in circularity, wall thickness, tube-to-tube distance, and intertube interactions. These factors are expected to cause inhomogeneous broadening of the scattered-light spectra and masking of effects occurring directly due to changes in wall thickness. Additionally, in the experiment, contributions from both the SPP and photonic modes are likely to contribute to the measured spectra. This finding underlines the importance of developing precise synthesis methods in conjunction with both spectroscopic studies of individual nanostructures and large area arrays to accurately characterize their optical properties.

Figures 3(c) and 3(d) show that the photonic mode peak electric-field intensity occurred at a nearly fixed wavelength with changing wall thickness. Therefore, it was concluded that the photonic mode does not contribute significantly to the apparent red shift and that the red shift occurs only for surface plasmon modes. Additionally, the large inner diameter suggested that interwall surface plasmon coupling (i.e. coupling between opposite walls of the same nanotube) was negligible. Moreover, the two simulated excitation schemes demonstrated that mode coupling and propagation was strongly dependent on incident excitation angle and polarization direction. These differences can be observed by comparing the radial electric-field intensity profiles for the two excitation schemes [Figs. 3(e) and 3(f)]. End-on excitation [Fig. 3(e)] resulted in a surface
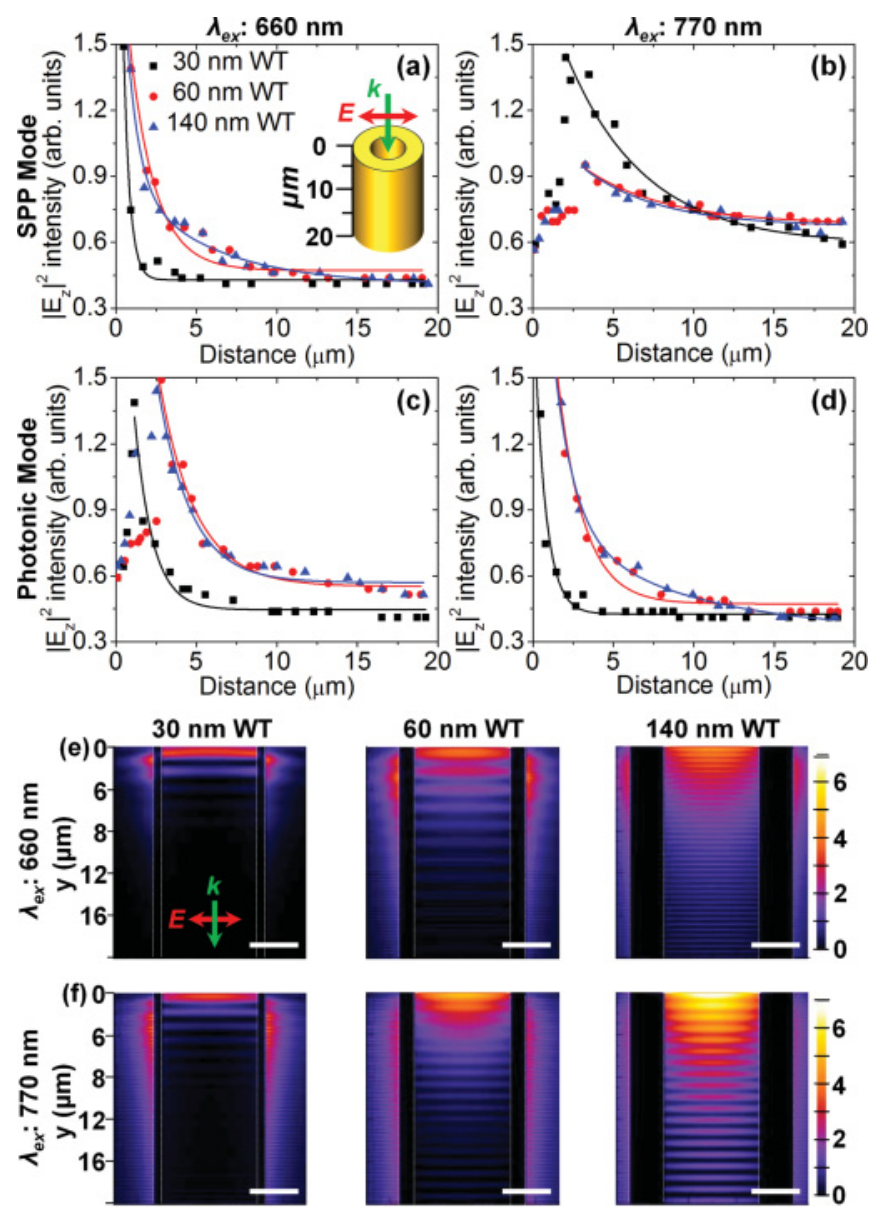

FIG. 4. (Color online) Simulated (a) and (b) SPP and (c) and (d) photonic mode decay profiles of single gold nanotubes under end-on excitation with constant 200-nm inner diameter and WT of 30,60, and $140 \mathrm{~nm}$ with an excitation wavelength of (a)-(c) $660 \mathrm{~nm}$ and (b)-(c) $770 \mathrm{~nm}$. Axial 2-D electric-field intensity profiles of the tube crosssection under (e) $660 \mathrm{~nm}$ and (f) $770 \mathrm{~nm}$ end-on excitation ( $x$-axis scale bar $=150 \mathrm{~nm}$; color bars represent electric field intensity). Inset in (a) is a schematic of the excitation conditions. Green/gray arrows indicate excitation direction $(k)$ and red/dark gray arrows indicate the polarization direction $(E)$.

plasmon mode on the outer surface of the AuNT, with little change in the shape of the electric-field intensity mode profile. Transversely polarized normal excitation [Fig. 3(f)] resulted in radial multipole surface plasmon resonances with electric-field intensity mode profiles that change with circumference, the nature of which will be discussed in the next section. Axially polarized normal excitation exhibited similar electric field profiles to those observed for end-on excitation.

To study how mode propagation is affected by wall thickness, SPP [Figs. 4(a) and 4(b)] and photonic [Figs. 4(c) and $4(\mathrm{~d})]$ mode propagation lengths were extracted from the simulated end-on excited electric-field intensity profiles [Figs. 4(e) and 4(f); nanotube length $=20 \mu \mathrm{m}$ ]. The propagation length of each mode was extracted by fitting with a single exponential decay function. Under $660 \mathrm{~nm}$ excitation, propagation lengths were determined to be $1.1 \pm 0.1,2.2 \pm$ 0.2 , and $2.0 \pm 0.2 \mu \mathrm{m}$ for the SPP modes and $0.4 \pm 0.03$, $1.2 \pm 0.1$, and $1.3 \pm 0.1 \mu \mathrm{m}$ for the photonic modes, for wall thicknesses of 30,60, and $140 \mathrm{~nm}$, respectively [Figs. 3(a) and 
3(c)]. Under $770 \mathrm{~nm}$ excitation, propagation lengths increased notably: $4.8 \pm 0.7,5.1 \pm 0.8$, and $4.7 \pm 1.6 \mu \mathrm{m}$ for the SPP modes and $0.7 \pm 0.1,1.6 \pm 0.1$, and $1.7 \pm 0.1 \mu \mathrm{m}$ for the photonic modes for wall thicknesses of 30,60 , and $140 \mathrm{~nm}$, respectively [Figs. 3(b) and 3(d)].

From these data, it was found that the SPP propagation length of the 30-nm WT AuNT under $660 \mathrm{~nm}$ excitation increased by a factor of 4.4 at the longer excitation wavelength $(770 \mathrm{~nm})$, whereas the SPP propagation lengths of the 60- and $140-\mathrm{nm}$ WTs only increased by a factor of $\sim 2.3$. In the case of the photonic mode, the propagation length increases with increasing wall thickness and longer excitation wavelengths, indicating that mode confinement is dependent on wall thickness. Unlike the change in SPP propagation length, the photonic mode propagation increases more uniformly across the three WTs at the longer wavelength (by factors of 1.75, 1.39 , and 1.30 for WTs 30, 60, and $140 \mathrm{~nm}$, respectively).

\section{DISCUSSION}

For plasmonic nanoparticles, the geometry dictates the modes that can be supported by the structure and, subsequently, the extinction peaks and spectral widths. Our experimental observations of the apparent red shift in the scattered-light spectra of AuNTs as a function of decreasing wall thicknesses are in qualitative agreement with simulation, in addition to prior literature on metallic tubular and core-shell structures. ${ }^{5-7,10}$ To further elucidate the results presented here and help attribute the observed experimental red shifts to particular mode types, it is necessary to place the present work in the context of prior work on metallic nanotubes and core-shell systems, which has primarily been concerned with smaller inner-diameter nanostructures.

Initial work towards understanding the fundamental mechanism that gives rise to the apparent red shift as a function of decreasing shell thickness in core-shell structures was conducted by Averitt et al. ${ }^{10,25}$ By adapting Mie scattering theory, the apparent red shift of gold nanoshells as a function of decreasing shell thickness was attributed purely to classical origins, dictated by the relative thickness of the shell and the core diameter. Broadening of the plasmon peak width was attributed to a reduced effective mean free path of the conduction electrons. It should be noted that the core-shell systems studied by Averitt et al. had shell thicknesses below the electron mean free path so electron surface scattering was important. ${ }^{10,11}$

The plasmon hybridization model of Prodan et al. offered further insight into the plasmonic response of tubular and coreshell nanostructures. ${ }^{1,11}$ This model, described as the electromagnetic analogue of molecular orbital theory, can predict the plasmonic responses of complex nanoparticle geometries in terms of a hybridization of plasmon modes arising from more elementary geometries. In particular, the model has been employed to describe the tunability of resonance frequencies in concentric multinanoshell structures as a function of intershell distance, shell thickness, and radius. Subsequently, Moradi built upon the plasmon hybridization model and adapted it specifically to tubular metallic nanostructures with concentric and nonconcentric cores. ${ }^{12,13,26}$ In this model, the plasmon resonances in metallic nanotubes can be described in terms of a hybridization of plasmon resonances in capillary and wire geometries. This hybridization results in the splitting of the plasmon resonances into a low-energy symmetric mode and a higher-energy antisymmetric mode. As the wall thickness of the nanotube decreases, the interaction and splitting between the two plasmon modes increases. ${ }^{13}$ This results in an effective decrease in energy of the symmetric mode and an increase in energy of the antisymmetric mode. This model is valid in a size regime where retardation effects are neglected and so the nanotubes described by the hybridization approach have diameters $<40 \mathrm{~nm} .{ }^{12,13}$ It is important to note that, in this size regime, interwall coupling is strong.

The nanotubes described in this paper all have radial dimensions approaching half a wavelength of visible light. Therefore, they are on a much larger length scale than the core-shell and tubular systems studied in the aforementioned works (i.e. $<40$-nm outer diameter). Thus, these AuNTs do not fall into the size regime where interwall plasmon mode coupling is strong or into the quasistatic size regime where retardation effects can be neglected. However, these large AuNTs do exhibit a characteristic red spectral shift with decreasing WT as observed in the scattered-light spectra of large area arrays (Fig. 1) and along the length of the NW/NT heterostrucutre (Fig. 2).

Therefore, we propose that the observed red shift in the experimental scattered-light data with decreasing wall thickness is due to the degree of coupling or hybridization between the core mode and the mode on the nanotube outer surface. As the wall thickness becomes optically thin, the mode in the core becomes leakier, allowing it to interact with the mode on the outer surface. In a variety of systems, ranging from chains of equidistant-spaced metal nanospheres (which exhibit with local surface plasmon modes), to films of metal separated by a dielectric layer IMI waveguides (which exhibit propagating SPP modes), increasing the spatial overlap between modes adjacent to the metal interface increases the degree of mode mixing or coupling, resulting in a red spectral shift in the lower energy mode. ${ }^{27-31}$

Mode coupling/hybridization in the AuNTs studied here is supported by the simulated SPP mode spectra and propagation length data as follows. While an increase in propagation length of the simulated SPP modes is expected at longer wavelengths for all wall thicknesses (due to reduced ohmic loss in the metal at longer wavelengths), the magnitude of enhancement in SPP mode propagation length at longer wavelengths was notably greater (by a factor of 4.4) for the thinnest WT $(30 \mathrm{~nm})$ compared to the thicker cases (60 and $140 \mathrm{~nm}$; propagation length increase of $\sim 2.3$ ); see Fig. 4. The greater propagation length enhancement for the thinnest AuNT wall thickness strongly suggests that increased coupling or hybridization between the propagating SPP mode on the outer surface of the AuNT and the mode in the core occurs, resulting in a lowering of the SPP mode energy [i.e. a red-shift; see Fig. 3(a)]. ${ }^{10,25,27-29}$

As previously noted, FDTD simulations of AuNTs under transversely polarized normal excitation support radial modes, with quality factors of up to eight at visible wavelengths. These modes are characteristic of ring-type surface plasmon resonances, similar in nature to that observed by Apter et al. at ultraviolet wavelengths. ${ }^{14}$ While these ring-resonant modes were not explicitly detected in our experiments due to the fact 
the scattered-light spectra were not conducted under a specific polarization direction, such resonances have been observed in low-aspect-ratio ring-shaped metallic nanostructures with quality factors ranging from 5-10. ${ }^{14,32-34}$

Apter et al. developed a nanoring-resonator model to describe high-energy plasmon resonances in tubular metallic nanoshells. ${ }^{14}$ The model treats the nanotube as an IMI structure that has been bent into an annular shape, i.e. a ring resonator, and is based on the dispersion relations of surface plasmons on planar IMI structures. ${ }^{14,35}$ This model predicts that the red shift in nanotube resonance wavelength is a function of increasing outer nanotube circumference in addition to wall thickness, a point which is not predicted by the hybridization model. Additionally, the ring resonator modes occur when the surface plasmon wavelength $\left(\lambda_{s p}\right)$ matches an integer fraction of the ring's effective perimeter $\left(P_{\text {eff }}=\pi\left(r_{1}+r_{2}\right)\right.$ where $r_{1}=\mathrm{ID} / 2$ and $r_{2}=r_{1}+\mathrm{WT}$ ) and can be calculated with the following equation: ${ }^{14}$

$$
\lambda_{s p}=P_{\text {eff }} / m
$$

where $m$ is the mode of the resonance $(m=1,2, \ldots)$. The calculated $\lambda_{s p}$ for AuNTs of 30-, 60-, and 140-nm WT with a fixed 200-nm ID are $722 \mathrm{~nm}(m=1), 816 \mathrm{~nm}(m=1)$, and $534 \mathrm{~nm}(m=2)$, respectively. These resonance wavelengths for the 30- and 140-nm WT AuNTs are within $8 \%$ of the simulated results [Fig. 3(b)], and for the 60-nm WT case, the value is within $15 \%$ of the simulated data. Therefore, it is highly likely that the large 200-nm ID AuNTs presented in this paper can support surface plasmon ring-resonances at visible wavelengths, in addition to propagating SPP modes.

In summary, gold nanotubes were fabricated via electrodeposition and selective pore widening of nanoporous alumina templates. Control over wall thickness was achieved by varying the pore-widening time. Scattered-light spectroscopy of nanotube arrays indicated that resonant scattering at visible wavelengths red shifts with decreasing nanotube wall thickness. Scattered-light spectra were recorded along a single nanowire/nanotube heterostructure to more accurately measure the apparent red shift without the inhomogenous broadening stemming from a distribution of gold nanotube wall thickness in the large area arrays. Finite-difference-timedomain simulations of gold nanotubes were conducted to understand the relationship between surface plasmon mode propagation and coupling as a function of wall thickness. Simulations show a red shift as a function of decreasing wall thickness for surface plasmon modes under both normal and end-on excitation directions. Additionally, a photonic mode was supported in the core of the nanotubes; however, its wavelength did not vary notably with wall thickness. Electric-field intensity profiles showed surface plasmon propagation analogous to insulator-metal-insulator waveguides under end-on and axially polarized normal excitation. Simulated gold nanotubes under transversely polarized normal excitation indicate their ability to support surface plasmon ring-resonator modes on the outer surface, which we plan to experimentally verify in future work using polarization-resolved dark-field spectroscopy measurements. With suitable choice of wall thickness, gold nanotubes may be employed for anisotropic light confinement and large-area arrays of vertically aligned waveguides or optical cavities.

\section{ACKNOWLEDGMENTS}

The authors thank Harry Atwater for support and useful discussions. J.K. acknowledges support from The Nanotechnology for Clean energy NSF IGERT Program. D.M.O'C. acknowledges support from the 7th European Community Framework Program (ACTOSPED project; PIOF-GA-2008221230) and the Institute for Advanced Materials, Devices and Nanotechnology at Rutgers University. M.F. acknowledges support from the Caltech SURF Program.
*Present address: Materials Department, University of California, Santa Barbara, California 93106, USA.

†ocarroll@ rutgers.edu

${ }^{1}$ E. Prodan and P. Norlander, Nano Lett. 3, 543 (2003).

${ }^{2}$ J. McPhillips, A. Murphy, M. P. Jonsson, W. R. Hendren, R. Atkison, F. Höök, A. W. Zayats, and R. J. Pollard, ACS Nano 4, 2210 (2010).

${ }^{3}$ S. J. Oldenburg, S. K. Westcott, R. D. Averitt, and N. J. Halas, J. Chem. Phys. 111, 4729 (1999).

${ }^{4}$ Y. Sun, B. Mayers, and Y. Xia, Adv. Mater. 15, 641 (2003).

${ }^{5}$ J. Zhu, Mat. Sci. Eng. A. 454-455, 685 (2007).

${ }^{6}$ A. Murphy and R. J. Pollard, 3rd International Congress on Advanced Electromagnetic Materials in Microwaves and Optics, London, 2009 (unpublished).

${ }^{7}$ A. Murphy, J. McPhillips, W. Hendren, C. McClatchey, R. Atkinson, G. Wurtz, A. V. Zayats, and R. Pollard, Nanotech. 22, 045705 (2011).

${ }^{8}$ C. E. Rayford II, G. Schatz, and K. Shuford, Nanoscape 2, 27 (2005).
${ }^{9}$ W. Zhang, S. H. Brongersma, O. Richard, B. Brijs, R. Palmans, L. Froyen, and K. Maex, Microelectron. Eng. 76, 146 (2004).

${ }^{10}$ R. D. Averitt, D. Sarkar, and N. J. Halas, Phys. Rev. Lett. 78, 4217 (1997).

${ }^{11}$ E. Prodan, C. Radloff, N. J. Halas, and P. Norlander, Science 302, 419 (2003).

${ }^{12}$ A. Moradi, J. Phys. Chem. Solids 69, 2936 (2008).

${ }^{13}$ A. Moradi, Physica B 405, 2466 (2010).

${ }^{14}$ B. Apter, O. Guilatt, and U. Efron, Appl. Opt. 50, 5457 (2011).

${ }^{15}$ J. J. Ju, S. Park, M. Kim, J. T. Kim, S. K. Park, Y. J. Park, and M. H. Lee, J. Lightwave Technol. 26, 0733 (2008).

${ }^{16}$ A. Boltasseva, T. Nikolajsen, K. Leosson, K. Kjaer, M. S. Larsen, and S. I. Bozhevolnyi, J. Lightwave Technol. 23, 413 (2005).

${ }^{17}$ R. Charbonneau, C. Scales, I. Breukelaar, S. Fafard, N. Lahoud, G. Mattiussi, and P. Berini, J. Lightwave Technol. 24, 477 (2006).

${ }^{18}$ R. Charbonneau, N. Lahoud, G. Mattiussi, and P. Berini, Opt. Exp. 13, 977 (2005).

${ }^{19}$ S. I. Bozhevolnyi, V. S. Volkov, E. Devaux, J. Laluet, and T. W. Ebbesen, Nature 440, 508 (2006). 
${ }^{20}$ A. Boltasseva and S. I. Bozhevolnyi, IEEE J. Sel. Topic. Quantum Electron. 12, 1233 (2006).

${ }^{21}$ Z. Han, L. Liu, and E. Forsberg, Opt. Commun. 259, 690 (2006).

${ }^{22}$ S. Park and S. H. Song, Electron. Lett. 42, 402 (2006).

${ }^{23}$ T. Nikolajsen, K. Leosson, and S. I. Bozhevolnyi, Appl. Phys. Lett. 88, 5833 (2004)

${ }^{24}$ S. J. Oldenburg, R. D. Averitt, S. L. Westcott, and N. J. Halas, Chem. Phys. Lett. 288, 243 (1998).

${ }^{25}$ R. D. Averitt, S. L. Westcott, and N. J. Halas, J. Opt. Soc. Am. B 16, 1824 (1999).

${ }^{26}$ A. Moradi, Opt. Commun. 282, 3368 (2009).

${ }^{27}$ A. O. Pinchuck and G. C. Schatz, Mater. Sci. Eng. B 149, 251 (2008).

${ }^{28}$ Y. H. Ye, Y. W. Jiang, M. W. Tsai, Y. T. Chang, C. Y. Chen, D. C. Tzuang, Y. T. Wu, and S. C. Lee, Appl. Phys. Lett. 93, 263106 (2008).
${ }^{29}$ R. Perahia, T. P. Mayer Alegre, A. H. Safavi-Naeini, and O. Painter, Appl. Phys. Lett. 95, 201114 (2009).

${ }^{30}$ R. Zia, M. D. Selker, P. B. Catrysse, and M. L. Brongersma, J. Opt. Soc. Am. A 21, 2442 (2004).

${ }^{31}$ J. A. Dionne, L. A. Sweatlock, and H. A. Atwater, Phys. Rev. B 72, 075405 (2005).

${ }^{32}$ J. Aizpurua, P. Hanarp, D. S. Sutherland, M. Käll, G. W. Bryant, and F. J. García de Abajo, Phys. Rev. Lett. 90, 057401-1 (2003).

${ }^{33}$ T. B. Wang, X. W. Wen, C. P. Yin, and H. Z. Wang, Opt. Express 17, 24096 (2009).

${ }^{34}$ A. Pors, M. Willatzen, O. Albrektsen, and S. I. Bozhevolnyi, J. Opt. Soc. Am. B 27, 1680 (2010).

${ }^{35}$ S. A. Maier, Plasmonics: Fundamentals and Applications (Springer, New York, 2007), p. 32. 